\title{
REMARK ON THE PERIODIC MASS CRITICAL NONLINEAR SCHRÖDINGER EQUATION
}

\author{
NOBU KISHIMOTO \\ (Communicated by James E. Colliander)
}

\begin{abstract}
We consider the mass critical NLS on $\mathbb{T}$ and $\mathbb{T}^{2}$. In the $\mathbb{R}^{d}$ case the Strichartz estimates enable us to show well-posedness of the IVP in $L^{2}$ (at least for small data) via the Picard iteration method. However, counterexamples to the $L^{6}$ Strichartz on $\mathbb{T}$ and the $L^{4}$ Strichartz on $\mathbb{T}^{2}$ were given by Bourgain (1993) and Takaoka-Tzvetkov (2001), respectively, which means that the Strichartz spaces are not suitable for iteration in these problems. In this note, we show a slightly stronger result, namely, that the IVP on $\mathbb{T}$ and $\mathbb{T}^{2}$ cannot have a smooth data-to-solution map in $L^{2}$ even for small initial data. The same results are also obtained for most of the two dimensional irrational tori.
\end{abstract}

\section{INTRODUCTION}

We consider the initial value problem of the mass critical nonlinear Schrödinger equation with periodic boundary condition:

$$
\left\{\begin{aligned}
i \partial_{t} u+\Delta u & =\mu|u|^{4 / d} u, \quad(t, x) \in \mathbb{R} \times \mathbb{T}^{d}, \\
u(0, x) & =u_{0}(x) \in L^{2}\left(\mathbb{T}^{d}\right) .
\end{aligned}\right.
$$

Here, $\mathbb{T}^{d}:=\mathbb{R}^{d} /(2 \pi \mathbb{Z})^{d}$ denotes the $d$ dimensional torus and $\mu= \pm 1$.

Concerning the initial value problem in $H^{s}\left(\mathbb{T}^{d}\right)$ in the case $d=1,2$, Bourgain [1] established the local well-posedness for $s>0$ via the Picard iteration method, and Christ, Colliander, Tao [6] showed the ill-posedness for $s<0$ in the sense that the data-to-solution map is not continuous as a map from $H^{s}(\mathbb{T})$ to even the space of distributions $\left(C^{\infty}(\mathbb{T})\right)^{*}$ if $s<0$. However, there is no result on the well-posedness of (1.1) in $L^{2}$ (neither positive nor negative), even for small data.

This difficulty comes from the lack of the periodic Strichartz estimate in the critical case 1 In fact, some counterexamples were given in the case of $d=1,2$ (namely, in the case that the nonlinearity $|u|^{4 / d} u$ is algebraic) as follows.

Received by the editors March 30, 2012 and, in revised form, July 24, 2012.

2010 Mathematics Subject Classification. Primary 35Q55; Secondary 46E35, 11P21.

Key words and phrases. Mass critical nonlinear Schrödinger equation, periodic boundary condition, Strichartz estimates, Picard iteration method.

This work was partially supported by Grant-in-Aid for Scientific Research 23840022.

${ }^{1}$ The energy critical defocusing NLS on $\mathbb{T}^{3}$ is known to be globally well-posed in the critical space $H^{1}\left(\mathbb{T}^{3}\right)$; see 1112 . In that case, trilinear Strichartz-type estimates with no loss of regularity (with respect to scaling) are available and play a crucial role in the proof, which is based on the Picard iteration. 
Theorem 1.1 (Bourgain [1], Takaoka-Tzvetkov [13]). The estimates

$$
\left\|e^{i t \partial_{x}^{2}} \phi\right\|_{L_{t, x}^{6}(\mathbb{T} \times \mathbb{T})} \lesssim\|\phi\|_{L^{2}(\mathbb{T})}
$$

and

$$
\left\|e^{i t \Delta} \phi\right\|_{L_{t, x}^{4}\left(\mathbb{T} \times \mathbb{T}^{2}\right)} \lesssim\|\phi\|_{L^{2}\left(\mathbb{T}^{2}\right)}
$$

do not hold.

In the case of $\mathbb{R}^{d}$, the corresponding Strichartz estimates hold and play an essential role in well-posedness theory at the critical regularity. In fact, one can easily show the small-data local well-posedness with a smooth data-to-solution map by the iteration argument using these Strichartz estimates. This is also true even for the $2 \mathrm{~d}$ semi-periodic case $\mathbb{R} \times \mathbb{T}\left[13\right.$. On $\mathbb{T}$ or $\mathbb{T}^{2}$, however, the above theorem suggests that the Strichartz space $L_{t, x}^{2+4 / d}\left(\mathbb{T}^{1+d}\right)$ is no longer appropriate for the resolution space when we try to apply the iteration. Then, a natural question is whether or not there is any other space suitable for iteration.

First, we will show that for $d=1,2$ the first nonlinear term in the Picard iteration scheme is not bounded in $L^{2}$.

Theorem 1.2. Let $d=1,2$ and $N, m \in \mathbb{N}$. Define $\phi_{m, N} \in L^{2}\left(\mathbb{T}^{d}\right)$ by

$$
\phi_{m, N}(x):=N^{-\frac{d}{2}} \sum_{k \in \mathbb{Z}_{N}^{d}} e^{i m k \cdot x},
$$

where $\mathbb{Z}_{N}^{d}:=\left\{\left(k_{1}, \ldots, k_{d}\right) \in \mathbb{Z}^{d}|| k_{j} \mid \leq N, 1 \leq j \leq d\right\}$. Then, we have $\left\|\phi_{m, N}\right\|_{L^{2}\left(\mathbb{T}^{d}\right)}$ $\sim 1$ and

$$
\left\|A\left[\phi_{m, N}\right]\left(\frac{2 \pi}{m^{2}}\right)\right\|_{L^{2}\left(\mathbb{T}^{d}\right)} \gtrsim \frac{1}{m^{2}} \log N
$$

where

$$
A[\phi](t):=-i \mu \int_{0}^{t} e^{i\left(t-t^{\prime}\right) \Delta}\left[\left|e^{i t^{\prime} \Delta} \phi\right|^{\frac{4}{d}} e^{i t^{\prime} \Delta} \phi\right] d t^{\prime} .
$$

The main result in this note is obtained as a corollary of the above theorem. This may be just a small but first step toward the expected goal of extending local well-posedness to $L^{2}$.

Corollary 1.3. Let $d=1,2$ and $T, r>0$ be arbitrarily small positive constants. Assume that the data-to-solution map $S: u_{0} \mapsto u(\cdot)$ associated with (1.1) on smooth data extends continuously to a map from the closed ball in $L^{2}\left(\mathbb{T}^{d}\right)$ of radius $r$ centered at the origin into $C\left([0, T] ; L^{2}\left(\mathbb{T}^{d}\right)\right)$. Then, this map will not be $C^{5}$ (resp. $C^{3}$ ) at the origin when $d=1$ (resp. $\left.d=2\right)$.

Results of this type were first mentioned by Bourgain [3] in the context of the $\mathrm{KdV}$ and the modified $\mathrm{KdV}$ initial value problems on $\mathbb{R}$ and $\mathbb{T}$. To prove Corollary [1.3, we assume it not to hold. Then, for $1 \mathrm{~d}$, the map $\phi \mapsto D^{5} S[\phi, \ldots, \phi](0)=$ $30 A[\phi]$ from $L^{2}(\mathbb{T})$ to $C\left([0, T] ; L^{2}(\mathbb{T})\right)$ would be continuous under the assumption of $C^{5}$. This contradicts Theorem 1.2 when we choose $m$ sufficiently large so that $\frac{2 \pi}{m^{2}} \leq T$. The same argument is applicable to $2 \mathrm{~d}$. 
Our result suggests that in the purely periodic setting the standard iteration argument, which would naturally give an analytic data-to-solution map, should fail to work for the mass critical NLS in $L^{2}$, even for small data. This is a big difference from the nonperiodic case.

We next take the spatial period into account and consider the torus $\mathbb{T}_{\alpha}^{d}:=$ $\mathbb{R}^{d} /\left(\alpha_{1} \mathbb{Z} \times \cdots \times \alpha_{d} \mathbb{Z}\right)$ with a general period $\alpha=\left(\alpha_{1}, \ldots, \alpha_{d}\right) \in \mathbb{R}_{+}^{d}$. Such consideration makes no sense when $d=1$, since a scaling argument allows us to normalize one component of $\alpha$. In two or higher dimensions, however, rationality of the torus may be of interest. We say the $2 \mathrm{~d}$ torus $\mathbb{T}_{\alpha}^{2}$ is rational (resp. irrational) if the ratio $\alpha_{2} / \alpha_{1}$ is (resp. is not) rational. We refer to [4,5] for the Strichartz estimates on general tori (both rational and irrational), which in general require a substantial amount of additional regularity.

It turns out that our argument for $d=2$ with a slight modification is applied not only to all of the rational tori but also to most of the irrational tori. To our knowledge, this is the first result concerning 2 d irrational tori at the $L^{2}$ regularity.

Theorem 1.4. Let $\gamma>0$ and $\mathbb{T}_{\gamma}^{2}:=\mathbb{R}^{2} /(2 \pi \mathbb{Z} \times 2 \pi \gamma \mathbb{Z})$. Suppose that $\gamma$ satisfies the following:

For any $\varepsilon>0$, there exist $p, q \in \mathbb{N}$ such that $\left|q^{2}-\frac{p^{2}}{\gamma^{2}}\right|<\varepsilon$.

Then, the $L^{4}$ Strichartz estimate

$$
\left\|e^{i t \Delta} \phi\right\|_{L_{t, x}^{4}\left([0, T] \times \mathbb{T}_{\gamma}^{2}\right)} \lesssim\|\phi\|_{L^{2}\left(\mathbb{T}_{\gamma}^{2}\right)}
$$

does not hold for any $T>0$. Moreover, the statement in Corollary 1.3 with $d=2$ and $\mathbb{T}^{2}$ replaced by $\mathbb{T}_{\gamma}^{2}$ holds. Furthermore, the set of all $\gamma>0$ not satisfying (1.4) is of Lebesgue measure zero.

Condition (1.4) means that $\gamma$ can be well approximated by a rational $\frac{p}{q}$. For functions on $\mathbb{T}_{\gamma}^{2}$ we consider the Fourier coefficients on the rescaled lattice $\mathbb{Z} \times \frac{1}{\gamma} \mathbb{Z}$. Then, if (1.4) is true, the sub-lattice $q \mathbb{Z} \times \frac{p}{\gamma} \mathbb{Z} \subset \mathbb{Z} \times \frac{1}{\gamma} \mathbb{Z}$ is close enough to a regular lattice $(q \mathbb{Z})^{2}$ so that a similar argument to the case of $\mathbb{T}^{2}$ can be applied. It seems, however, that the restriction (1.4) is just a technical one.

Related to the $L^{6}$ Strichartz estimate (1.2), a similar estimate for the Airy equation,

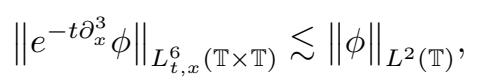

has been attracting attention. This estimate itself is open so far, while Bourgain [2] proved it with $\varepsilon$-loss of regularity and conjectured that it would be true.

This note is organized as follows. In the next section, we give a proof of Theorem 1.2. As a by-product of the proof, in Section 3 we show Theorem 1.1 in a different way without using the Weyl sum approach. These proofs are modified in Section 4 to verify Theorem 1.4. In the last section, we make some remarks on the $L^{6}$ Strichartz estimate for the Airy equation. 


\section{Proof of Theorem 1.2}

First, we give a proof for the $2 \mathrm{~d}$ case.

Proof of Theorem $1.2, d=2$. Since

$$
e^{i t \Delta} \phi_{m, N}=N^{-1} \sum_{k \in \mathbb{Z}_{N}^{2}} e^{-i m^{2}|k|^{2} t} e^{i m k \cdot x},
$$

we have

$$
\begin{aligned}
A\left[\phi_{m, N}\right](t, x)= & c N^{-3} \sum_{\substack{k \in \mathbb{Z}_{3 N}^{2}\\
}} e^{i m k \cdot x} \int_{0}^{t} e^{-i m^{2}|k|^{2}\left(t-t^{\prime}\right)} \\
& \times \sum_{\substack{k_{1}, k_{2}, k_{3} \in \mathbb{Z}_{N}^{2} \\
k_{1}-k_{2}+k_{3}=k}} e^{-i m^{2}\left(\left|k_{1}\right|^{2}-\left|k_{2}\right|^{2}+\left|k_{3}\right|^{2}\right) t^{\prime}} d t^{\prime} .
\end{aligned}
$$

Therefore, for $k \in \mathbb{Z}_{3 N}^{2}$,

$$
\begin{aligned}
\hat{A}\left[\phi_{m, N}\right]\left(\frac{2 \pi}{m^{2}}, m k\right) & =c N^{-3} \sum_{\substack{k_{1}, k_{2}, k_{3} \in \mathbb{Z}_{N}^{2} \\
k_{1}-k_{2}+k_{3}=k}} \int_{0}^{2 \pi / m^{2}} e^{-i m^{2}\left(\left|k_{1}\right|^{2}-\left|k_{2}\right|^{2}+\left|k_{3}\right|^{2}-|k|^{2}\right) t^{\prime}} d t^{\prime} \\
& =c m^{-2} N^{-3} \# \Gamma(k),
\end{aligned}
$$

where the set of all resonant interactions $\Gamma(k)$ is defined as

$(2.1) \Gamma(k):=\left\{\left.\left(k_{1}, k_{2}, k_{3}\right) \in\left(\mathbb{Z}_{N}^{2}\right)^{3}\left|k_{1}-k_{2}+k_{3}=k,\right| k_{1}\right|^{2}-\left|k_{2}\right|^{2}+\left|k_{3}\right|^{2}=|k|^{2}\right\}$.

This kind of set was previously observed in 9 . In particular, the conditions for $\left(k_{1}, k_{2}, k_{3}\right)$ to be in $\Gamma(k)$ is equivalent to the condition that four segments $\overline{k k_{1}}, \overline{k_{1} k_{2}}$, $\overline{k_{2} k_{3}}, \overline{k_{3} k}$ form a rectangle (possibly degenerate); see [9], section 2.2, for details.

It suffices to prove

$$
\# \Gamma(k) \gtrsim N^{2} \log N \quad \text { for } k \in \mathbb{Z}_{N / 2}^{2}
$$

By translation, we may assume $k=0$. The estimate (2.2) then follows from the next lemma.

Lemma 2.1. We have

$$
\#\left\{\left(k_{1}, k_{3}\right) \in\left(\mathbb{Z}_{N}^{2}\right)^{2} \mid k_{1} \cdot k_{3}=0\right\} \gtrsim N^{2} \log N .
$$

Proof. It suffices to count the number of elements of

$$
\left\{(p, q, r, s) \in \mathbb{Z}^{4} \mid 0<q \leq p \leq N, 0<s \leq N, p r+q s=0\right\},
$$

but this is exactly equal to 2

$$
\sum_{\substack{0<q \leq p \leq N \\ \operatorname{gcd}(p, q)=1}}\left[\frac{N}{p}\right]^{2}=\sum_{p=1}^{N}\left[\frac{N}{p}\right]^{2} \varphi(p) \sim N^{2} \sum_{p=1}^{N} \frac{\varphi(p)}{p^{2}},
$$

\footnotetext{
${ }^{2}$ There is a one-to-one correspondence between all the possible 'directions' of vectors in $\mathbb{Z}_{+}^{2}$ and all $(p, q) \in \mathbb{Z}_{+}^{2}$ with $p$ co-prime to $q$. Also, there are exactly $[N / \max \{p, q\}]$ points in $\mathbb{Z}_{N}^{2} \cap \mathbb{Z}_{+}^{2}$ for each direction $(p, q)$.
} 
where $[a]$ denotes the greatest integer not greater than $a$ and $\varphi(p)$ is Euler's totient function (that is, the number of positive integers not greater than and relatively prime to $p$ ). Recalling the identity $\sum_{d \mid n} \varphi(d)=n 3$ we have 4

$$
\log (N+1)<\sum_{n=1}^{N} \frac{1}{n}=\sum_{n=1}^{N} \frac{1}{n^{2}} \sum_{\substack{1 \leq p, l \leq n \\ p l=n}} \varphi(p) \leq \sum_{p, l=1}^{N} \frac{\varphi(p)}{p^{2} l^{2}}<\frac{\pi^{2}}{6} \sum_{p=1}^{N} \frac{\varphi(p)}{p^{2}},
$$

obtaining the claim.

Proof for the $1 \mathrm{~d}$ quintic case is reduced to the above $2 \mathrm{~d}$ result.

Proof of Theorem $1.2, d=1$. Similarly to the case $d=2$, we have

$$
\begin{aligned}
\hat{A}\left[\phi_{m, N}\right]\left(\frac{2 \pi}{m^{2}}, m k\right) & =c N^{-\frac{5}{2}} \sum_{\substack{k_{1}, k_{2}, k_{3}, k_{4}, k_{5} \in \mathbb{Z}_{N} \\
k_{1}-k_{2}+k_{3}-k_{4}+k_{5}=k}} \int_{0}^{2 \pi / m^{2}} e^{-i m^{2}\left(k_{1}^{2}-k_{2}^{2}+k_{3}^{2}-k_{4}^{2}+k_{5}^{2}-k^{2}\right) t^{\prime}} d t^{\prime} \\
& =c m^{-2} N^{-\frac{5}{2}} \# \Gamma^{\prime}(k),
\end{aligned}
$$

where

$$
\begin{aligned}
\Gamma^{\prime}(k):=\left\{\left(k_{1}, k_{2}, k_{3}, k_{4}, k_{5}\right) \in \mathbb{Z}_{N}^{5} \mid\right. & k_{1}-k_{2}+k_{3}-k_{4}+k_{5}=k, \\
& \left.k_{1}^{2}-k_{2}^{2}+k_{3}^{2}-k_{4}^{2}+k_{5}^{2}=k^{2}\right\},
\end{aligned}
$$

and it suffices to show

$$
\# \Gamma^{\prime}(k) \gtrsim N^{2} \log N \quad \text { for } k \in \mathbb{Z}_{N / 2} .
$$

Putting

$$
\begin{aligned}
& l_{1}=\frac{k_{1}+k_{2}}{2}, n_{1}=\frac{k_{1}-k_{2}}{2}, l_{2}=\frac{k_{3}+k_{4}}{2}, \\
& n_{2}=\frac{k_{3}-k_{4}}{2}, l_{3}=\frac{k_{5}+k}{2}, n_{3}=\frac{k_{5}-k}{2},
\end{aligned}
$$

we reduce (2.3) to

$\#\left\{\left(l_{1}, \ldots, n_{3}\right) \in \mathbb{Z}_{N}^{6} \mid n_{1}+n_{2}+n_{3}=0, l_{1} n_{1}+l_{2} n_{2}+l_{3} n_{3}=0, l_{3}=n_{3}+k\right\} \gtrsim N^{2} \log N$,

which is further simplified to

$\#\left\{\left(l_{1}, l_{2}, n_{1}, n_{2}\right) \in \mathbb{Z}_{N}^{4} \mid\left(l_{1}+n_{1}+n_{2}-k\right) n_{1}+\left(l_{2}+n_{1}+n_{2}-k\right) n_{2}=0\right\} \gtrsim N^{2} \log N$.

Then, denoting $l_{j}^{\prime}:=l_{j}+n_{1}+n_{2}-k(j=1,2)$, it suffices to show that

$$
\#\left\{\left(l_{1}^{\prime}, l_{2}^{\prime}, n_{1}, n_{2}\right) \in \mathbb{Z}_{N}^{4} \mid l_{1}^{\prime} n_{1}+l_{2}^{\prime} n_{2}=0\right\} \gtrsim N^{2} \log N .
$$

This is nothing but Lemma 2.1 .

\footnotetext{
${ }^{3}$ This identity follows from the fact that $\#\left\{k \mid 1 \leq k \leq n, \operatorname{gcd}(k, n)=\frac{n}{d}\right\}=\phi(d)$ for each positive divisor $d$ of $n$, which is a consequence of the equivalence $\operatorname{gcd}(m, n)=p \Leftrightarrow \operatorname{gcd}(m / p, n / p)=1$.

${ }^{4}$ Indeed, we have $\lim _{N \rightarrow \infty} \frac{1}{\log N} \sum_{p=1}^{N} \frac{\varphi(p)}{p^{2}}=\frac{6}{\pi^{2}}$. This is the limiting case of the identity $\sum_{p=1}^{\infty} \frac{\varphi(p)}{p^{s}}=$ $\frac{\zeta(s-1)}{\zeta(s)}$ for $s>2$, where $\zeta(s):=\sum_{p=1}^{\infty} \frac{1}{p^{s}}$.
} 


\section{Proof of Theorem 1.1}

Here, we give another proof of the failure of (1.3) and (1.2). We use $\phi=\phi_{1, N}$ for the sequence of data breaking these estimates. There is no difference between $1 \mathrm{~d}$ and $2 \mathrm{~d}$, so we focus on the $2 \mathrm{~d}$ estimate (1.3). The exact calculation shows that

$$
\begin{aligned}
& \left\|e^{i t \Delta} \phi_{1, N}\right\|_{L_{t, x}^{4}\left(\mathbb{T}^{3}\right)}^{4} \sum_{k_{1}, k_{2}, k_{3}, k_{4} \in \mathbb{Z}_{N}^{2}} \int_{\mathbb{T}^{3}} e^{-i\left(\left|k_{1}\right|^{2}-\left|k_{2}\right|^{2}+\left|k_{3}\right|^{2}-\left|k_{4}\right|^{2}\right) t} e^{i\left(k_{1}-k_{2}+k_{3}-k_{4}\right) \cdot x} d x d t \\
& =(2 \pi)^{3} N^{-4} \#\left\{\left(k_{1}, k_{2}, k_{3}, k_{4}\right) \in\left(\mathbb{Z}_{N}^{2}\right)^{4} \mid k_{1}-k_{2}+k_{3}-k_{4}=0,\right. \\
& \geq(2 \pi)^{3} N^{-4} \sum_{k_{4} \in \mathbb{Z}_{N / 2}^{2}} \# \Gamma\left(k_{4}\right) \\
& \gtrsim \log N,
\end{aligned}
$$

where $\Gamma(k)$ is as in (2.1) and we have used (2.2) in the last inequality. Since $\left\|\phi_{1, N}\right\|_{L^{2}\left(\mathbb{T}^{2}\right)} \sim 1$, the estimate (1.3) fails for sufficiently large $N$. This choice of initial data is the same as 13 , but we obtain the lower bound $(\log N)^{1 / 4}$, which is better than $(\log \log N)^{1 / 4}$ in [13. For (1.2) we can show the same lower bound $(\log N)^{1 / 6}$ as in $[1$.

We can also disprove

$$
\left\|e^{i t \Delta} \phi\right\|_{L_{t, x}^{2+d / 2}\left([0, T] \times \mathbb{T}^{d}\right)} \lesssim\|\phi\|_{L^{2}\left(\mathbb{T}^{d}\right)}
$$

for arbitrary $T>0(d=1,2)$ by choosing $m \in \mathbb{N}$ so that $\frac{2 \pi}{m^{2}} \leq T$ and repeating the above argument with $\phi=\phi_{m, N}$.

In the remainder of this section, let us consider the $L^{4}$ estimate on $\mathbb{T} \times \mathbb{T}^{3}$ :

$$
\left\|e^{i t \Delta} \phi\right\|_{L_{t, x}^{4}\left(\mathbb{T} \times \mathbb{T}^{3}\right)} \lesssim N^{\frac{1}{4}}\|\phi\|_{L^{2}\left(\mathbb{T}^{3}\right)}
$$

for all $\phi \in L^{2}$ with $\hat{\phi}(k) \equiv 0$ if $|k|>N$. Bourgain [1] proved (3.1) up to an $\varepsilon$ loss of regularity, but (3.1) itself has been open. We will see that the same sequence of data $\phi_{1, N}$ does not break it any longer, so one cannot disprove (3.1) by just adapting $1 \mathrm{~d}$ or $2 \mathrm{~d}$ counterexample to the $3 \mathrm{~d}$ situation.

Recalling that $\phi_{1, N}(x):=\frac{1}{N^{3 / 2}} \sum_{k \in \mathbb{Z}_{N}^{3}} e^{i k \cdot x}$, we have

$$
\begin{gathered}
\left\|e^{i t \Delta} \phi_{1, N}\right\|_{L_{t, x}^{4}\left(\mathbb{T}^{4}\right)}^{4}=(2 \pi)^{4} N^{-6} \sum_{k_{4} \in \mathbb{Z}_{N}^{3}} \# \Gamma^{\prime \prime}\left(k_{4}\right), \\
\Gamma^{\prime \prime}\left(k_{4}\right):=\left\{\left.\left(k_{1}, k_{2}, k_{3}\right) \in\left(\mathbb{Z}_{N}^{3}\right)^{3}\left|k_{1}-k_{2}+k_{3}=k_{4},\right| k_{1}\right|^{2}-\left|k_{2}\right|^{2}+\left|k_{3}\right|^{2}=\left|k_{4}\right|^{2}\right\} .
\end{gathered}
$$

We will show $\# \Gamma^{\prime \prime}(k) \lesssim N^{4}$ for any $k \in \mathbb{Z}_{N}^{3}$, which verifies that $\phi_{1, N}$ obeys (3.1) for all $N$. Similar to the 2 d case we observed in the previous section, the rectangular structure of resonant frequencies implies that it suffices to prove $\# \Gamma^{\prime \prime}(0) \lesssim N^{4}$, and that $\# \Gamma^{\prime \prime}(0) \leq \#\left\{\left(k_{1}, k_{3}\right) \in\left(\mathbb{Z}_{N}^{3}\right)^{2} \mid k_{1} \cdot k_{3}=0\right\}$. 
In the case that either $k_{1}$ or $k_{3}$ is located on an axis, we easily obtain a bound of $O\left(N^{3}\right)$. For instance, if $k_{1} \in \mathbb{Z}_{N} \times\{(0,0)\}$, then $k_{3}$ must be in $\{0\} \times \mathbb{Z}_{N}^{2}$.

Hence, without loss of generality, we count the number of $\left(k_{1}, k_{3}\right)$ such that $k_{1} \in\left\{(x, y, z) \in \mathbb{Z}^{3} \mid 0<z \leq y \leq x \leq N\right\}$. For any 'direction' $(a, b, c)(0<c \leq$ $b \leq a \leq N$ and $\operatorname{gcd}(a, b, c)=1)$, there are exactly $\left[\frac{N}{a}\right]$ choices of $k_{1}$ facing in that direction, and $k_{3}$ must be perpendicular to that direction for such a $k_{1}$. Therefore, the number of possible $\left(k_{1}, k_{3}\right)$ is bounded by

$$
\sum_{\substack{0<c \leq b \leq a \leq N \\ \operatorname{gcd}(a, b, c)=1}} \frac{N}{a} \#\left(\mathcal{P}_{(a, b, c)} \cap \mathbb{Z}_{N}^{3}\right),
$$

where $\mathcal{P}_{(a, b, c)}$ is the plane in $\mathbb{R}^{3}$ including the origin and is perpendicular to $(a, b, c)$. Then, it suffices to prove $(3.2)=O\left(N^{4}\right)$.

Fix $(a, b, c)$ satisfying the above condition and set $n:=\operatorname{gcd}(a, b),(a, b)=$ $\left(n a^{\prime}, n b^{\prime}\right)$. Then,

$$
\#\left(\mathcal{P}_{(a, b, c)} \cap \mathbb{Z}_{N}^{3} \cap\left\{(x, y, z) \in \mathbb{Z}^{3} \mid z=0\right\}\right) \sim \frac{N}{a^{\prime}}
$$

Since $\operatorname{gcd}(c, n)=1$, the plane $\left\{(x, y, z) \in \mathbb{Z}^{3} \mid z=l\right\}$ is possible to intersect $\mathcal{P}_{(a, b, c)} \cap \mathbb{Z}_{N}^{3}$ if and only if $l$ is a multiple of $n$. Therefore, we have

$$
\#\left(\mathcal{P}_{(a, b, c)} \cap \mathbb{Z}_{N}^{3}\right) \sim \frac{N}{a^{\prime}} \cdot \frac{N}{n}=\frac{N^{2}}{a}
$$

and then

$$
\begin{aligned}
& \sum_{\substack{0<c \leq b \leq a \leq N \\
\operatorname{gcd}(a, b, c)=1}} \frac{N}{a} \#\left(\mathcal{P}_{(a, b, c)} \cap \mathbb{Z}_{N}^{3}\right) \\
& \quad \sim N^{3} \sum_{a=1}^{N} \frac{1}{a^{2}} \#\{(b, c) \mid 0<c \leq b \leq a, \operatorname{gcd}(a, b, c)=1\} .
\end{aligned}
$$

We also have

$$
\begin{aligned}
& \#\{(b, c) \mid 0<c \leq b \leq a, \operatorname{gcd}(a, b, c)=1\}=\sum_{\substack{1 \leq n \leq a \\
n \mid a}} \sum_{\substack{1 \leq b \leq a \\
\operatorname{gcd}(b, a)=n}} \sum_{\substack{1 \leq c \leq b \\
\operatorname{gcd}(c, n)=1}} 1 \\
& =\sum_{\substack{1 \leq n \leq a \\
n \mid a}} \sum_{\substack{1 \leq b^{\prime} \leq a / n \\
\operatorname{gcd}\left(b^{\prime}, a / n\right)=1}} b^{\prime}(n) \leq \sum_{\substack{1 \leq n \leq a \\
n \mid a}} \sum_{\substack{1 \leq b^{\prime} \leq a / n \\
\operatorname{gcd}\left(b^{\prime}, a / n\right)=1}} \frac{a}{n} \cdot n=a \sum_{\substack{1 \leq n \leq a \\
n \mid a}} \varphi\left(\frac{a}{n}\right)=a^{2},
\end{aligned}
$$

where in the last equality we have used that $\sum_{n \mid a} \varphi\left(\frac{a}{n}\right)=\sum_{n \mid a} \varphi(n)=a$. Plugging this into (3.3) we obtain (3.2) $\lesssim N^{4}$, as desired. 


\section{The Case of general tori}

Let us give a proof of Theorem 1.4 for a general 2 d torus $\mathbb{T}_{\gamma}^{2}:=\mathbb{R}^{2} /(2 \pi \mathbb{Z} \times 2 \pi \gamma \mathbb{Z})$ $(\gamma>0)$. Under condition (1.4), for any $N \gg 1$ we can choose $p, q \in \mathbb{N}$ such that

$$
\left|q^{2}-\frac{p^{2}}{\gamma^{2}}\right|<\frac{1}{N^{2}}, \quad q>N .
$$

Indeed, it is trivial if $\gamma \in \mathbb{Q}$, and otherwise it suffices to take $p, q$ given in (1.4) with

$$
\varepsilon=\min \left\{\frac{1}{N^{2}}, \min _{p, q \in \mathbb{N}, q \leq N}\left|q^{2}-\frac{p^{2}}{\gamma^{2}}\right|\right\}>0 .
$$

Using such $(p, q)$ we define $\phi_{N}: \mathbb{T}_{\gamma}^{2} \rightarrow \mathbb{C}$ by

$$
\phi_{N}(x, y):=\frac{1}{N} \sum_{k=\left(k_{x}, k_{y}\right) \in \mathbb{Z}_{N}^{2}} e^{i\left(q k_{x} x+\frac{p}{\gamma} k_{y} y\right)},
$$

which has the size of $O(1)$ in $L^{2}\left(\mathbb{T}_{\gamma}^{2}\right)$. Similarly to Section 2 , we have

$$
\hat{A}\left[\phi_{N}\right]\left(t, q k_{x}, \frac{p}{\gamma} k_{y}\right)=c e^{-i\left(q^{2} k_{x}^{2}+\frac{p^{2}}{\gamma^{2}} k_{y}^{2}\right) t} N^{-3} \sum_{\substack{k_{1}, k_{2}, k_{3} \in \mathbb{Z}_{N}^{2} \\ k_{1}-k_{2}+k_{3}=k}} \int_{0}^{t} e^{-i \Phi t^{\prime}} d t^{\prime}
$$

for $k=\left(k_{x}, k_{y}\right) \in \mathbb{Z}^{2}$, where

$$
\begin{aligned}
\Phi & :=q^{2}\left(k_{1, x}^{2}-k_{2, x}^{2}+k_{3, x}^{2}-k_{x}^{2}\right)+\frac{p^{2}}{\gamma^{2}}\left(k_{1, y}^{2}-k_{2, y}^{2}+k_{3, y}^{2}-k_{y}^{2}\right) \\
& =q^{2}\left(\left|k_{1}\right|^{2}-\left|k_{2}\right|^{2}+\left|k_{3}\right|^{2}-|k|^{2}\right)-\left(q^{2}-\frac{p^{2}}{\gamma^{2}}\right)\left(k_{1, y}^{2}-k_{2, y}^{2}+k_{3, y}^{2}-k_{y}^{2}\right) .
\end{aligned}
$$

We split $A\left[\phi_{N}\right]$ into the resonant and nonresonant parts:

$$
\begin{aligned}
& \hat{A}\left[\phi_{N}\right]\left(t, q k_{x}, \frac{p}{\gamma} k_{y}\right) \\
& =c e^{-i\left(q^{2} k_{x}^{2}+\frac{p^{2}}{\gamma^{2}} k_{y}^{2}\right) t} N^{-3}\left(\sum_{\left|k_{1}\right|^{2}-\left|k_{2}\right|^{2}+\left|k_{3}\right|^{2}=|k|^{2}}+\sum_{\left|k_{1}\right|^{2}-\left|k_{2}\right|^{2}+\left|k_{3}\right|^{2} \neq|k|^{2}}\right) \int_{0}^{t} e^{-i \Phi t^{\prime}} d t^{\prime} \\
& =: \hat{A}_{\text {res }}\left[\phi_{N}\right]\left(t, q k_{x}, \frac{p}{\gamma} k_{y}\right)+\hat{A}_{\text {nonres }}\left[\phi_{N}\right]\left(t, q k_{x}, \frac{p}{\gamma} k_{y}\right) .
\end{aligned}
$$

For the resonant part, we have

$$
|\Phi|=\left|\left(q^{2}-\frac{p^{2}}{\gamma^{2}}\right)\left(k_{1, y}^{2}-k_{2, y}^{2}+k_{3, y}^{2}-k_{y}^{2}\right)\right| \lesssim \frac{1}{N^{2}} \cdot N^{2}=1,
$$

which implies $\Re \int_{0}^{t} e^{-i \Phi t^{\prime}} d t^{\prime} \geq \frac{t}{2}$ for any $0<t \ll 1$. Therefore, from (2.2) we have

$$
\left|\hat{A}_{r e s}\left[\phi_{N}\right]\left(t, q k_{x}, \frac{p}{\gamma} k_{y}\right)\right| \gtrsim t N^{-1} \log N
$$

for any $k \in \mathbb{Z}_{N / 2}^{2}$ and $0<t \ll 1$. For the nonresonant part, it holds that

$$
\begin{aligned}
|\Phi| & \geq\left|q^{2}\left(\left|k_{1}\right|^{2}-\left|k_{2}\right|^{2}+\left|k_{3}\right|^{2}-|k|^{2}\right)\right|-\left|\left(q^{2}-\frac{p^{2}}{\gamma^{2}}\right)\left(k_{1, y}^{2}-k_{2, y}^{2}+k_{3, y}^{2}-k_{y}^{2}\right)\right| \\
& \geq q^{2}-O(1) \gtrsim N^{2},
\end{aligned}
$$


which implies $\left|\int_{0}^{t} e^{-i \Phi t^{\prime}} d t^{\prime}\right| \lesssim N^{-2}$ for any $t \in \mathbb{R}$. Therefore,

$$
\left|\hat{A}_{\text {nonres }}\left[\phi_{N}\right]\left(t, q k_{x}, \frac{p}{\gamma} k_{y}\right)\right| \lesssim N^{-3} \cdot N^{4} \cdot N^{-2}=N^{-1}
$$

Consequently, we have

$$
\left\|A\left[\phi_{N}\right](t)\right\|_{L^{2}\left(\mathbb{T}_{\gamma}^{2}\right)} \gtrsim t \log N
$$

for any $(\log N)^{-1} \ll t \ll 1$, which shows that the map $\phi \mapsto A[\phi](t)$ on $L^{2}$ is not continuous at the origin for any $0<t \ll 1$. From this we deduce the same conclusion as Corollary 1.3. The failure of the $L^{4}$ Strichartz estimate is shown in a similar manner; we refer to Section 3 and omit the proof.

Finally, we observe condition (1.4), which is equivalent to the following:

$$
\text { For any } \varepsilon>0 \text {, there exist } p, q \in \mathbb{N} \text { such that }\left|\gamma-\frac{p}{q}\right|<\frac{\varepsilon}{q^{2}} \text {. }
$$

This is satisfied if $\gamma \in \mathbb{Q}$, so we may assume $\gamma \notin \mathbb{Q}$. Recall the continued fraction expansion of $\gamma$ (cf. [10], Chapter X): There exists a unique sequence of positive integers $\left\{a_{n}\right\}_{n=1}^{\infty}$ such that $\gamma$ is the limit of the sequence of finite continued fractions

$$
[\gamma]+\frac{1}{a_{1}+\frac{1}{a_{2}+\frac{1}{\ddots+\frac{1}{a_{n}}}}}, \quad n=1,2, \ldots,
$$

as $n \rightarrow \infty$. It is known (10, section 11.10) that (4.1) will be satisfied if the sequence $\left\{a_{n}\right\}$ corresponding to $\gamma$ is unbounded 5 and that the set of $\gamma$ for which $\left\{a_{n}\right\}$ is bounded is null. Hence, almost every $\gamma>0$ satisfies (1.4).

\section{Note on the $L^{6}$ Strichartz estimate FOR THE AIRY EQUATION}

The $L^{6}$ Strichartz estimate for the Airy equation,

$$
\left\|e^{-t \partial_{x}^{3}} \phi\right\|_{L_{t, x}^{6}(\mathbb{T} \times \mathbb{T})} \lesssim\|\phi\|_{L^{2}(\mathbb{T})},
$$

is a challenging open problem proposed by Bourgain [2], who proved instead

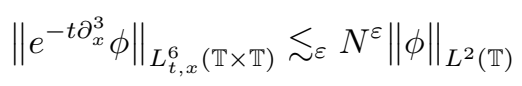

for all $\phi \in L^{2}$ with $\hat{\phi}(k) \equiv 0$ if $|k|>N$.

\footnotetext{
${ }^{5}$ In fact, 4.1 holds if and only if $\left\{a_{n}\right\}$ is unbounded. To see this, let $p_{n}, q_{n} \in \mathbb{N}$ be such that $\frac{p_{n}}{q_{n}}$ is the irreducible fraction representation of (4.2). Then, it holds that under the convention $q_{0}=1$,

$$
\frac{1}{q_{n}^{2} a_{n+1}}>\left|\gamma-\frac{p_{n}}{q_{n}}\right|>\frac{1}{q_{n}^{2}\left(a_{n+1}+2\right)}>\frac{1}{q_{n-1}^{2}\left(a_{n}+1\right)^{2}\left(a_{n+1}+2\right)}
$$

for any $n$. Moreover, the sequence $\left\{\frac{p_{n}}{q_{n}}\right\}$ is the best rational approximation of $\gamma$ in the sense that

$$
\left|\gamma-\frac{p_{n}}{q_{n}}\right|=\min \left\{\left|\gamma-\frac{p}{q}\right| \mid p, q \in \mathbb{N}, q \leq q_{n}\right\}
$$

for any $n$ (see [10], Theorem 181). Hence, if $a_{n} \leq M<\infty$ for all $n$, we have

$$
\left|\gamma-\frac{p}{q}\right|>\frac{1}{(M+1)^{2}(M+2) q^{2}}
$$

for any $p, q \in \mathbb{N}$. As a corollary, it turns out that no quadratic irrational $\gamma$ (i.e., an irrational root of a quadratic equation with integral coefficients) satisfies (1.4), since $\left\{a_{n}\right\}$ for such an irrational is periodic (see [10, Theorem 177).
} 
Let us recall the proof of (5.2). Let $\phi=\sum_{k \in \mathbb{Z}_{N}} a_{k} e^{i k x}$. By the Cauchy-Schwarz inequality,

$$
\begin{aligned}
& \left\|e^{-t \partial_{x}^{3}} \phi\right\|_{L_{t, x}^{6}\left(\mathbb{T}^{2}\right)}^{6}=\left\|\sum_{k_{1}, k_{2}, k_{3} \in \mathbb{Z}_{N}} a_{k_{1}} a_{k_{2}} a_{k_{3}} e^{i\left(k_{1}^{3}+k_{2}^{3}+k_{3}^{3}\right) t} e^{i\left(k_{1}+k_{2}+k_{3}\right) x}\right\|_{L_{t, x}^{2}\left(\mathbb{T}^{2}\right)}^{2} \\
& =\sum_{n, k \in \mathbb{Z}}\left|\sum_{\substack{k_{1}, k_{2}, k_{3} \in \mathbb{Z}_{N} \\
k_{1}+k_{2}+k_{3}=k, k_{1}^{3}+k_{2}^{3}+k_{3}^{3}=n}} a_{k_{1}} a_{k_{2}} a_{k_{3}}\right|^{2} \\
& \leq \sum_{k \in \mathbb{Z}_{N}}\left|3 \sum_{k_{1} \in \mathbb{Z}_{N}} a_{k_{1}} a_{-k_{1}} a_{k}\right|^{2} \\
& +\left(\sup _{\substack{n \in \mathbb{Z}_{3 N^{3}}, k \in \mathbb{Z}_{3 N} \\
n \neq k^{3}}} \# \Gamma_{\text {Airy }}(n, k)\right) \\
& \times \sum_{n, k \in \mathbb{Z}} \sum_{\substack{k_{1}, k_{2}, k_{3} \in \mathbb{Z}_{N} \\
k_{1}+k_{2}+k_{3}=k, k_{1}^{3}+k_{2}^{3}+k_{3}^{3}=n}}\left|a_{k_{1}}\right|^{2}\left|a_{k_{2}}\right|^{2}\left|a_{k_{3}}\right|^{2} \\
& \leq 9\|\phi\|_{L^{2}(\mathbb{T})}^{6}+\|\phi\|_{L^{2}(\mathbb{T})}^{6} \sup _{\substack{n \in \mathbb{Z}_{3 N^{3}}, k \in \mathbb{Z}_{3 N} \\
n \neq k^{3}}} \# \Gamma_{\text {Airy }}(n, k),
\end{aligned}
$$

where

$$
\Gamma_{\text {Airy }}(n, k):=\left\{\left(k_{1}, k_{2}, k_{3}\right) \in \mathbb{Z}_{N}^{3} \mid k_{1}+k_{2}+k_{3}=k, k_{1}^{3}+k_{2}^{3}+k_{3}^{3}=n\right\} .
$$

Some divisor-counting argument then yields the bound

$$
\# \Gamma_{\text {Airy }}(n, k) \lesssim e^{\frac{c \log N}{\log \log N}} \lesssim \varepsilon N^{\varepsilon} \quad \text { for }|n| \lesssim N^{3} \text { and }|k| \lesssim N \text { s.t. } n \neq k^{3},
$$

which implies (5.2).

The above proof says that we could establish (5.1) if we had the uniform estimate

$$
\# \Gamma_{\text {Airy }}(n, k) \lesssim 1 \quad \text { for } n, k \in \mathbb{Z} \text { s.t. } n \neq k^{3} .
$$

A similar estimate holds if we put some further restriction on the set $\Gamma_{\text {Airy }}(n, k)$. In fact, Colliander et al. 8] proved (5.3) with $\Gamma_{\text {Airy }}(n, k)$ replaced by

$$
\begin{aligned}
\Gamma_{\text {Airy }}^{\prime}(n, k):=\left\{\left(k_{1}, k_{2}, k_{3}\right) \in \Gamma_{\text {Airy }}(n, k)|| k_{\text {max }}|\gg| k_{\text {med }}|\gg| k_{\text {min }} \mid,\right. & \\
& \left.\left|k_{\text {max }}\right| \gg\left|k_{\text {min }}\right|^{3}\right\},
\end{aligned}
$$

where $k_{\text {max }}, k_{\text {med }}, k_{\text {min }}$ are the maximum, the median, and the minimum among $k_{1}, k_{2}, k_{3}$, respectively, and used it in 7 to prove the global well-posedness of the Korteweg-de Vries equation on $\mathbb{T}$ at the limiting regularity $H^{-1 / 2}$.

Unfortunately, (5.3) itself is false 6 More precisely, it holds that for each $N \gg 1$ there exists $k \in \mathbb{Z}$ such that $|k| \lesssim N$ and

$$
\# \Gamma_{\text {Airy }}\left(\frac{k^{3}}{9}, k\right) \gtrsim \log N
$$

\footnotetext{
${ }^{6}$ The author could not find any article pointing out this fact.
} 
To see this, we first notice that $k_{1}+k_{2}+k_{3}=k$ and $k_{1}^{3}+k_{2}^{3}+k_{3}^{3}=n$ imply

$$
\begin{gathered}
\left(k-k_{1}\right)+\left(k-k_{2}\right)+\left(k-k_{3}\right)=2 k, \\
\left(k-k_{1}\right)\left(k-k_{2}\right)\left(k-k_{3}\right)=\frac{1}{3}\left\{\left(k_{1}+k_{2}+k_{3}\right)^{3}-\left(k_{1}^{3}+k_{2}^{3}+k_{3}^{3}\right)\right\}=\frac{k^{3}-n}{3} .
\end{gathered}
$$

Putting $k_{j}^{\prime}:=\frac{1}{2}\left(k-k_{j}\right)(j=1,2,3)$, we consider sets of three integers $k_{1}^{\prime}, k_{2}^{\prime}, k_{3}^{\prime}$ which have common sum and product. Now, consider three rational numbers

$$
\frac{(x+1)^{2}}{x}, \quad-\frac{x^{2}}{x+1}, \quad-\frac{1}{x(x+1)}
$$

for some $x \in \mathbb{N}$. Note that the sum and the product of these three numbers are 3 and 1, respectively, independent of $x 7$ Moreover, a different choice of $x$ gives a different set of three numbers, since these three fractions are all irreducible. Hence, for an arbitrary $m \in \mathbb{N}$ we find $m$ sets of three integers

$$
\left(\frac{(x+1)^{2}}{x} M, \quad-\frac{x^{2}}{x+1} M, \quad-\frac{1}{x(x+1)} M\right), \quad x \in\{1,2, \ldots, m\},
$$

with the common sum $3 M$ and the common product $M^{3}$, where $M$ denotes the least common multiple 8 of $1,2, \ldots, m+1$. It is easily verified that

$$
\log M=\log \prod_{\substack{p: \text { prime } \\ p \leq m+1}} p^{\left[\frac{\log (m+1)}{\log p}\right]} \leq \pi(m+1) \log (m+1),
$$

where $\pi(n)$ is the number of prime numbers not greater than $n$. From the prime number theorem 9 we obtain $\log M \lesssim m$ for large $m$. Finally, from $2 \cdot 3 M=2 k$ and $8 \cdot M^{3}=\frac{1}{3}\left(k^{3}-n\right)$ we have $k=3 M$ and $n=3 M^{3}$, and we find $m$ sets of three integers

$$
\left(\left(3-\frac{2(x+1)^{2}}{x}\right) M, \quad\left(3+\frac{2 x^{2}}{x+1}\right) M, \quad\left(3+\frac{2}{x(x+1)}\right) M\right), \quad x \in\{1,2, \ldots, m\},
$$

with the common sum $3 M$ and the common cubic sum $3 M^{3}$.

We remark that the above observation does not seem strong enough to disprove (5.1), because the logarithmic growth is verified only for very few $(n, k)$. Conversely, (5.1) could be established if we had

$$
\sum_{k \in \mathbb{Z}}\left|\sum_{\substack{k_{1}, k_{2}, k_{3} \in \mathbb{Z}_{N} \\ k_{1}+k_{2}+k_{3}=k, k_{1}^{3}+k_{2}^{3}+k_{3}^{3}=k^{3} / 9}} a_{k_{1}} a_{k_{2}} a_{k_{3}}\right|^{2} \lesssim\|\phi\|_{L^{2}(\mathbb{T})}^{6}
$$

and

$$
\# \Gamma_{\text {Airy }}(n, k) \lesssim 1 \quad \text { for } n, k \in \mathbb{Z} \text { s.t. } n \neq k^{3} \text { and } n \neq \frac{k^{3}}{9} .
$$

It is not clear, however, whether both or either of them is true.

\footnotetext{
${ }^{7}$ This is actually true for three fractions $\frac{x^{2}}{y z}, \frac{y^{2}}{z x}, \frac{z^{2}}{x y}$ with $x, y, z \in \mathbb{Z}$ satisfying $x+y+z=0$ and $x y z \neq 0$. The above one is just a special case $(y=1, z=-x-1)$ of it.

${ }^{8}$ Since $x+1$ is relatively prime to $x, M / x(x+1) \in \mathbb{Z}$.

${ }^{9} \pi(n) \sim \frac{n}{\log n}(n \gg 1)$.
} 


\section{REFERENCES}

[1] J. Bourgain, Fourier transform restriction phenomena for certain lattice subsets and applications to nonlinear evolution equations. I. Schrödinger equations, Geom. Funct. Anal. 3 (1993), no. 2, 107-156, DOI 10.1007/BF01896020. MR1209299 (95d:35160a)

[2] J. Bourgain, Fourier transform restriction phenomena for certain lattice subsets and applications to nonlinear evolution equations. II. The KdV-equation, Geom. Funct. Anal. 3 (1993), no. 3, 209-262, DOI 10.1007/BF01895688. MR1215780(95d:35160b)

[3] J. Bourgain, Periodic Korteweg de Vries equation with measures as initial data, Selecta Math. (N.S.) 3 (1997), no. 2, 115-159, DOI 10.1007/s000290050008. MR1466164(2000i:35173)

[4] J. Bourgain, On Strichartz's inequalities and the nonlinear Schrödinger equation on irrational tori, Mathematical aspects of nonlinear dispersive equations, Ann. of Math. Stud., vol. 163, Princeton Univ. Press, Princeton, NJ, 2007, pp. 1-20. MR2331676 (2008j:35165)

[5] F. Catoire and W.-M. Wang, Bounds on Sobolev norms for the defocusing nonlinear Schrödinger equation on general flat tori, Commun. Pure Appl. Anal. 9 (2010), no. 2, 483491, DOI 10.3934/cpaa.2010.9.483. MR2600446 (2011h:35270)

[6] M. Christ, J. Colliander, and T. Tao, Instability of the periodic nonlinear Schrödinger equation, preprint (2003). arXiv:0311227

[7] J. Colliander, M. Keel, G. Staffilani, H. Takaoka, and T. Tao, Sharp global well-posedness for $K d V$ and modified $K d V$ on $\mathbb{R}$ and $\mathbb{T}$, J. Amer. Math. Soc. 16 (2003), no. 3, 705-749 (electronic), DOI 10.1090/S0894-0347-03-00421-1. MR1969209 (2004c:35352)

[8] J. Colliander, M. Keel, G. Staffilani, H. Takaoka, and T. Tao, Multilinear estimates for periodic KdV equations, and applications, J. Funct. Anal. 211 (2004), no. 1, 173-218, DOI 10.1016/S0022-1236(03)00218-0. MR2054622 (2005a:35241)

[9] J. Colliander, M. Keel, G. Staffilani, H. Takaoka, and T. Tao, Transfer of energy to high frequencies in the cubic defocusing nonlinear Schrödinger equation, Invent. Math. 181 (2010), no. 1, 39-113, DOI 10.1007/s00222-010-0242-2. MR2651381 (2011f:35320)

[10] G. H. Hardy and E. M. Wright, An introduction to the theory of numbers, 6th ed., revised by D. R. Heath-Brown and J. H. Silverman. With a foreword by Andrew Wiles. Oxford University Press, Oxford, 2008. MR2445243 (2009i:11001)

[11] Sebastian Herr, Daniel Tataru, and Nikolay Tzvetkov, Global well-posedness of the energycritical nonlinear Schrödinger equation with small initial data in $H^{1}\left(\mathbb{T}^{3}\right)$, Duke Math. J. 159 (2011), no. 2, 329-349, DOI 10.1215/00127094-1415889. MR2824485 (2012j:35392)

[12] Alexandru D. Ionescu and Benoit Pausader, The energy-critical defocusing NLS on $\mathbb{T}^{3}$, Duke Math. J. 161 (2012), no. 8, 1581-1612, DOI 10.1215/00127094-1593335. MR2931275

[13] H. Takaoka and N. Tzvetkov, On 2D nonlinear Schrödinger equations with data on $\mathbb{R} \times \mathbb{T}$, J. Funct. Anal. 182 (2001), no. 2, 427-442, DOI 10.1006/jfan.2000.3732. MR 1828800 (2002c:35251)

Department of Mathematics, Kyoto University, Kitashirakawa-Oiwakecho, Sakyo, KYOTO 606-8502, JAPAN

Current address: Research Institute for Mathematical Sciences, Kyoto University, Kitashirakawa-Oiwakecho, Sakyo, Kyoto 606-8502, Japan

E-mail address: nobu@kurims.kyoto-u.ac.jp 